\title{
Overweight and obesity may be a risk factor for thyroid dysfunction in pregnant women: a systematic review and meta-analysis
}

\author{
E. Nalbant-Poyraz ${ }^{1}$ and J. Farebrother ${ }^{1}$ \\ ${ }^{1}$ Human Nutrition Laboratory, Institute of Food, Nutrition and Health, ETH Zurich, Switzerland
}

Obesity is associated with hypothyroidism ${ }^{(1)}$. Adequate iodine intake and thyroid function are essential for normal foetal development ${ }^{(2)}$ Women with a body mass index (BMI) above normal who become pregnant may have an increased risk of thyroid dysfunction during gestation. To assess this risk, we reviewed the thyroid function of pregnant women (PW) with overweight or obesity compared to those with normal weight.

Following the Preferred Reporting Items for Systematic Reviews and Meta-Analyses guidelines ${ }^{(3)}$ we searched PubMed, Web of Science and Epistemonikos from inception to 21.07.2020. We included published articles written in English, in populations of any iodine status and describing intervention and observational studies that reported thyroid stimulating hormone (TSH) and thyroid hormone concentrations in PW with BMI stratification. We assessed quality of studies using the Joanna Briggs Institute Critical Appraisal Checklist for Studies Reporting Prevalence Data ${ }^{(4)}$ extracted medians and percentiles, and used the quantile estimation method to elaborate underlying distributions of study subgroups. We calculated the difference of medians (DM) with $95 \%$ confidence intervals $(95 \% \mathrm{CI})$ between normal weight and overweight or obese or both. We pooled results with a random-effects meta-analysis and plotted our findings using RStudio ${ }^{(5)}$.

From a search yield of 1354 articles, we included 7 studies ( 2 cohort, 2 cross-sectional, 1 retrospective cross-sectional, 1 prospective follow-up, 1 prospective observational study) with 20,572 subjects. No data from intervention trials were retrieved. In trimester one, PW with overweight and obesity had higher TSH (DM: $0.10 \mathrm{mIU} / \mathrm{L} ; 95 \% \mathrm{CI}: 0.02,0.19 ; \mathrm{I} 2: 60.2 \% ; \mathrm{P}=0.012 ; \mathrm{n}=7$ studies) and lower free thyroxine (fT4) (DM: $-0.60 \mathrm{pmol} / \mathrm{L} ; 95 \% \mathrm{CI}:-0.80,-0.36 ; \mathrm{I} 2: 71.5 \% ; \mathrm{P}<0.001 ; \mathrm{n}=7$ studies). We found lower fT4 in trimesters two (DM: $-0.43 \mathrm{pmol} / \mathrm{L} ; 95 \% \mathrm{CI}:-0.74,-0.12 ; \mathrm{I} 2: 44.5 \% ; \mathrm{P}=0.005 ; \mathrm{n}=2$ studies) and three (DM: $-0.40 \mathrm{pmol} / \mathrm{L} ; 95 \% \mathrm{CI}:-0.75,-0.04$; I2: $53.4 \% ; P=0.024 ; n=2$ studies) in PW with overweight or obesity, though TSH remained unchanged. Iodine intake (urinary iodine concentration, UIC) and historical prevalence data retrieved from literature suggested generally adequate iodine intakes except for one site with a UIC $<$ WHO threshold.

An increased TSH DM and lowered fT4 DM in the first trimester and lowered fT4 DM in second and third trimesters may imply a greater risk for hypothyroidism and isolated hypothyroxinemia in PW with overweight and obesity. This meta-analysis is limited by the low number of included studies with high heterogeneity. Further, most studies did not test for habitual iodine intakes. Yet, these findings highlight the lack of literature, particularly randomized controlled trials, that investigate the effect of interventions including iodine supplementation or fortification, on thyroid hormones in PW with overweight or obesity. Given the rising prevalence of obesity globally, supporting these women through the vulnerable life stage of pregnancy is a research priority.

\section{References}

1. Song R, Wang B, Yao Q, et al. (2019) J Front Immunol 10

2. Korevaar T, Medici M, Visser TJ, et al. (2017) Nat Rev Endocrinol 13(10), 610-622.

3. Moher D, Liberati A, Tetzlaff J, et al. (2009) BMJ 339, b2535.

4. Munn Z, MClinSc SM, Lisy K, et al. (2015) Int J Evid Based Health 13(3), 147-153.

5. McGrath S, Sohn H, Steele R, et al. (2020) Biom J 62, 69-98. 